\title{
EFECTIVIDAD DE UNA INTERVENCIÓN EDUCATIVA DE ENFERMERÍA SOBRE LA MODIFICACIÓN DE FACTORES DE RIESGO CORONARIOS ${ }^{1}$
}

\section{EDUCATIONAL EFFECTIVENESS OF NURSING INTERVENTION ON THE MODIFICATION OF RISK FACTORS CORONARY}

\author{
IVONNE BREVIS URRUTIA * \\ Sandra Valenzuela Suazo ${ }^{* *}$ \\ Katia SÁez Carrillo ${ }^{* * *}$
}

\begin{abstract}
RESUMEN
Este estudio se realizó con el propósito de modificar los factores de riesgo coronario de un grupo de pacientes que padecieron un infarto agudo al miocardio, realizándoles una intervención educativa de enfermería orientada a la Rehabilitación Cardiovascular. Objetivo: Evaluar la efectividad de la intervención educativa a este grupo de pacientes. Material y método: Estudio de tipo cuasi-experimental, realizado en la Unidad Cardioquirúrgica del Hospital Clínico Regional de Concepción, de enero a septiembre del 2012, que presentaban múltiples factores de riesgo coronarios, los cuales fueron sometidos a un régimen de ejercicios físicos, así como sesiones educativas. La muestra fue de 63 pacientes, el grupo control quedo constituido por 32 pacientes y el grupo experimental por 31, a ambos grupos se les aplicó una encuesta biosociodemográfica. Resultados: Se observó que los factores de riesgo coronario: obesidad, sedentarismo, hábito de fumar, e hipertensión arterial disminuyeron significativamente. Conclusión: La realización de esta intervención educativa orientada a la rehabilitación cardiovascular, cumple un papel valioso en la modificación de los factores de riesgo coronario, lo que disminuiría la morbimortalidad por esta enfermedad.
\end{abstract}

Palabras clave: Infarto del miocardio, estudios de intervención, enfermería cardiovascular.

\begin{abstract}
This study was performed in order to modify coronary risk factors in a group of patients who suffered an acute myocardial infarction performing a nursing educational intervention oriented at Cardiovascular Rehabilitation. Objective: To assess the effectiveness of the educational intervention in this group of patients. Methods: Quasiexperimental Study, conducted in the cardiac surgery unit of the Regional Hospital of Concepción, from January to September 2012, which had multiple coronary risk factors, which were subject to a system of physical exercises, as well as educational sessions. Sample consisted of 63 patients, the control group consisting of 32 patients and 31 for the experimental group, both groups were given a biosociodemographic survey. Results: It

\footnotetext{
${ }^{1}$ Estudio extraído de Tesis de Doctorado "Intervención de enfermería orientada al cuidado durante la rehabilitación cardiovascular en pacientes post infarto agudo al miocardio”, Programa de Doctorado, Departamento de Enfermería, Universidad de Concepción, Chile.

*Enfermera. Unidad de Emergencia Hospital Guillermo Grant Benavente, Concepción, Chile. Email: ibrevis@gmail.com

${ }^{* *}$ Enfermera. Profesora Departamento de Enfermería, Universidad de Concepción, Chile. Email: svalenzu@udec.cl

${ }^{* * *}$ Estadística. Profesora Departamento Estadística. Universidad de Concepción, Chile. Email: ksaez@udec.cl
} 
was found that coronary risk factors: obesity, sedentary lifestyle, smoking, and hypertension were significantly reduced. Conclusion: The realization of this cardiovascular rehabilitation oriented educational intervention plays a valuable role for coronary risk factors modification, which would decrease the morbidity and mortality from this disease.

Key words: Myocardial infarction, intervention studies, cardiovascular nursing.

Fecha recepción: 04/04/13. Fecha aceptación: 20/11/14.

\section{INTRODUCCIÓN}

Las enfermedades cardiovasculares constituyen la primera causa de muerte en pacientes adultos en la mayoría de los países desarrollados, y se espera que también lo sean en los países en vías de desarrollo en el año 2020 (1). Dentro de éstas, la manifestación más dramática es el infarto agudo al miocardio (IAM), al que sobreviven, al cabo de un año, sólo la mitad de los pacientes, muchos de los cuales no se recuperan en forma óptima, siendo una entidad de alta prevalencia en el mundo occidental, incluido Chile (2).

La valoración de los factores de riesgo (FR) se ha convertido en una preocupación importante del equipo de salud. Este término fue utilizado por primera vez en el estudio clásico de Framingham (3) y se define como una condición biológica, de estilo de vida, socioeconómica, que se halla asociada con un incremento de la probabilidad de enfermar. Los principales FR que se asocian con la enfermedad coronaria son: edad, género, hipercolesterolemia, hipertrigliceridemia, hipertensión arterial, tabaquismo, diabetes mellitus, presencia de enfermedad coronaria, antecedentes familiares de enfermedad coronaria, obesidad y sedentarismo. En Chile, la encuesta de vida saludable patrocinada por el Ministerio de Salud da muestra de la crítica situación que se vive, con índices de sedentarismo de $88,6 \%$, exceso de peso $67 \%$, tabaquismo $40,6 \%$, dislipidemia $38,5 \%$, hipertensión arterial 26,9\%, diabetes 9,4\% (4,
5). En el incremento de los FR se observa un marcado matiz metabólico, que aumentará la incidencia de Enfermedades Cardiovasculares (ECV) en los próximos años (6).

En pacientes que sobreviven a un IAM, la Rehabilitación Cardiovascular (RC) es fundamental, ya que, asociado al entrenamiento físico complementado con otras medidas de prevención como la educación, pueden reducir la mortalidad total y cardiovascular en un $20-25 \%$ (7). Los primeros datos sobre la RC se encuentran tras la publicación de los informes de la Organización Mundial de la Salud en el año1964, donde se define como (8): "Un conjunto de actividades necesarias para asegurar a los pacientes coronarios una condición física, mental y social óptima, por medio del cual puedan reintegrarse por sus propios medios a la vida familiar y profesional". Esta definición incluye algo más que la actividad física, ya que introduce la calidad de vida y la readaptación a un estatus familiar, social y profesional, al menos, igual al que tenían antes del evento coronario. Esta readaptación debe basarse en: mejor conocimiento de la enfermedad, movilización precoz, para evitar el deterioro físico y las complicaciones del reposo prolongado, entrenamiento físico correcto, equilibrio psicológico adecuado y prevención secundaria de los FR.

Los programas de RC incluyen pautas de actividad física, de apoyo y fortalecimiento psicológico y control de FR, éstos pueden determinarse a corto y a largo plazo. A corto plazo: la readaptación física para reanudar paulatinamente las actividades diarias, edu- 
cación a pacientes y familia sobre el proceso de la enfermedad y apoyo psicológico durante la fase de recuperación; a largo plazo: la identificación y el tratamiento de los FR que influyen en la progresión de la enfermedad, reforzamiento de comportamientos sanos que mejoren el pronóstico, la situación óptima de la capacidad física y facilitar el regreso a las actividades laborales y profesionales. La $\mathrm{RC}$ se divide en 3 fases, las que buscan estabilizar la enfermedad y evitar su progresión: Fase Hospitalaria (fase I), Fase de Convalecencia (fase II) y la Fase de Mantenimiento (fase III). Estas fases serán diferentes según las características de cada paciente, y vienen determinadas por el tipo de cardiopatía, sus complicaciones y el resultado de las pruebas complementarias.

Se hace necesario apoyar a estos pacientes a través de la incorporación de intervenciones de salud desde una perspectiva integral, que potencien su rehabilitación y les permitan adaptarse a su nuevo estado de ser enfermos coronarios, lo que les facilitará sobrellevar mejor su enfermedad y reducir las complicaciones asociadas. El profesional de enfermería es quien permanece más tiempo en contacto directo y cara a cara con él o la paciente, convirtiéndose en el pilar fundamental de la RC; cumple una labor destacada en la educación de éste como enfatizando la importancia y el significado de los FR coronarios.

En el estudio de Winslow y Weber (9), se aplicó un programa de educación sobre modificación de factores de riesgo a 60 pacientes coronarios y a sus familias, cinco días previos al alta, con el fin de minimizar el desacondicionamiento, mejorar la función cardiaca, proporcionar una actividad de formación progresiva y asegurar un nivel de actividad apropiada al alta; se enseñaron temas como el IAM y angina, factores de riesgo coronarios, medicamentos, dieta y vuelta al hogar. Este grupo se comparó con 60 pacientes, grupo control que no participó en el programa educativo. Se encontró un aumento signifi- cativo en el conocimiento de los pacientes después de asistir al programa educativo. Por otra parte, Scalzi et al. (10) estudiaron durante dos años a 32 pacientes con un primer episodio de IAM, con el fin de investigar la relación entre la participación en un programa educacional organizado y la adherencia a las instrucciones médicas relacionadas con medicamentos, actividad física, consumo de cigarrillos, reanudar la actividad sexual, retorno al trabajo, consumo de alcohol, reducción de peso y ajuste emocional. Se realizaron mediciones al $1^{\circ}, 3^{\circ}, 6^{\circ}, 12^{\circ}, 18^{\circ}$ y $24^{\circ}$ meses posalta. Se concluye que la retención de información es limitada durante la fase aguda de la enfermedad, pero la instrucción continua en visitas posteriores parece mejorar el conocimiento y la adherencia.

En la población chilena no se encuentran reportes en la literatura respecto a una intervención enfermería para la RC con énfasis en la educación en pacientes que han sufrido un IAM, y de algún seguimiento posterior a su egreso hospitalario.

El objetivo fue evaluar la efectividad de una intervención educativa de enfermería orientada al cuidado de enfermería en la rehabilitación cardiovascular de pacientes posinfarto agudo al miocardio, en comparación con aquellos pacientes a los que no se les aplica la intervención, sino que reciben las indicaciones rutinarias que se dan al alta donde se encuentran hospitalizados.

\section{MATERIAL Y MÉTODO}

Estudio cuantitativo, de tipo cuasiexperimental, que contó con dos grupos: un grupo experimental y un grupo control. La muestra quedo conformada por los pacientes que ingresaron a la Unidad de Cardiocirugía del Hospital Guillermo Grant Benavente Concepción, con diagnóstico de IAM, durante el período comprendido desde enero a septiembre 2012, que cumplieran los criterios de 
inclusión: pacientes de ambos sexos, entre 35 a 90 años, primer episodio de IAM, pacientes que hablen castellano y que sepan leer y escribir, pacientes que vivan en la Región del Bío-Bío. Criterios de exclusión: pacientes con algún tipo de limitación sensorial (visión, audición), pacientes con antecedentes de enfermedad mental, pacientes con hospitalización prolongada, pacientes que presenten complicaciones derivadas del IAM.

El tamaño de la muestra se fijó, a partir de lo planteado sobre aspectos a considerar, del tamaño mínimo de una muestra para estudios cuantitativos (11). Para estudios de tipo cuasiexperimental, el tamaño mínimo indicado es de 15 casos (12), por lo tanto, considerando el número de pacientes que ingresa con diagnóstico de IAM (323 pacientes durante el año 2011), se estimó una muestra de 60 pacientes, 30 pacientes para grupo control y 30 pacientes para grupo experimental, quedando la muestra total conformada por 63 pacientes con diagnóstico de IAM (31 en el grupo experimental y 32 en el grupo control). Durante el estudio no hubo pérdida de casos.

El tipo de muestreo fue no probabilístico, ya que se desconoce la probabilidad que posee cada caso de ser incluido en la muestra, y la selección se realizó mediante métodos en los que no interviene el azar, ya que se realizó en la medida que los pacientes iban ingresando a la Unidad, primeras dos semanas del mes grupo control, y dos semanas siguientes grupo experimental. De esta forma se evitó que los grupos se mezclaran y se generara sesgo y se aseguró que fuera representativa de la población en estudio. Se utilizó la técnica de muestreo consecutivo, que consistió en seleccionar a los pacientes que cumplieron los criterios de selección, a medida que ingresaron durante el período de tiempo determinado para la investigación. Al grupo experimental se le aplicó una intervención educativa orientada al cuidado de enfermería en la RC, y al grupo control sólo se le aplicó la educación habitual que otorga la Unidad de Cardiocirugía a los pacientes una vez que son dados de alta.

Para la recolección de datos se realizó una entrevista motivacional, un análisis de documentos (ficha clínica y de enfermería), lo que se registró en un instrumento recolector de datos biosociodemográficos y clínicos, donde se registro datos clínicos, de tratamiento y de las mediciones que se le realizó a cada paciente en forma individualizada (peso, talla, circunferencia de cintura, presión arterial $y$ frecuencia cardiaca), previo al alta, al mes y a los 3 meses de haber egresado del Servicio Clínico, todo esto a través de una visita domiciliaria. Esta visita domiciliaria significó, desde el punto de vista de la intervención y en función de las necesidades del paciente: conocer el hogar, el entorno y la situación familiar; detectar necesidades, recursos y redes; evaluar a la familia como apoyo en el cuidado; mejorar la definición del problema de salud. Permitió visualizar a la persona, no como una patología sino como un ser humano que tiene un problema de salud; y considerando que la principal labor del profesional de enfermería es lograr que cambien su conducta y adopten hábitos más saludables. Es fundamental esta instancia, ya que se logra una interacción más directa con el paciente, hay más confianza y se logra obtener mayor información en relación a lo que el paciente necesita; por lo que se puede otorgar al paciente las herramientas necesarias para su cuidado.

A continuación se presenta el Modelo de Intervención de Enfermería para la rehabilitación de pacientes con IAM, con énfasis en la educación. 


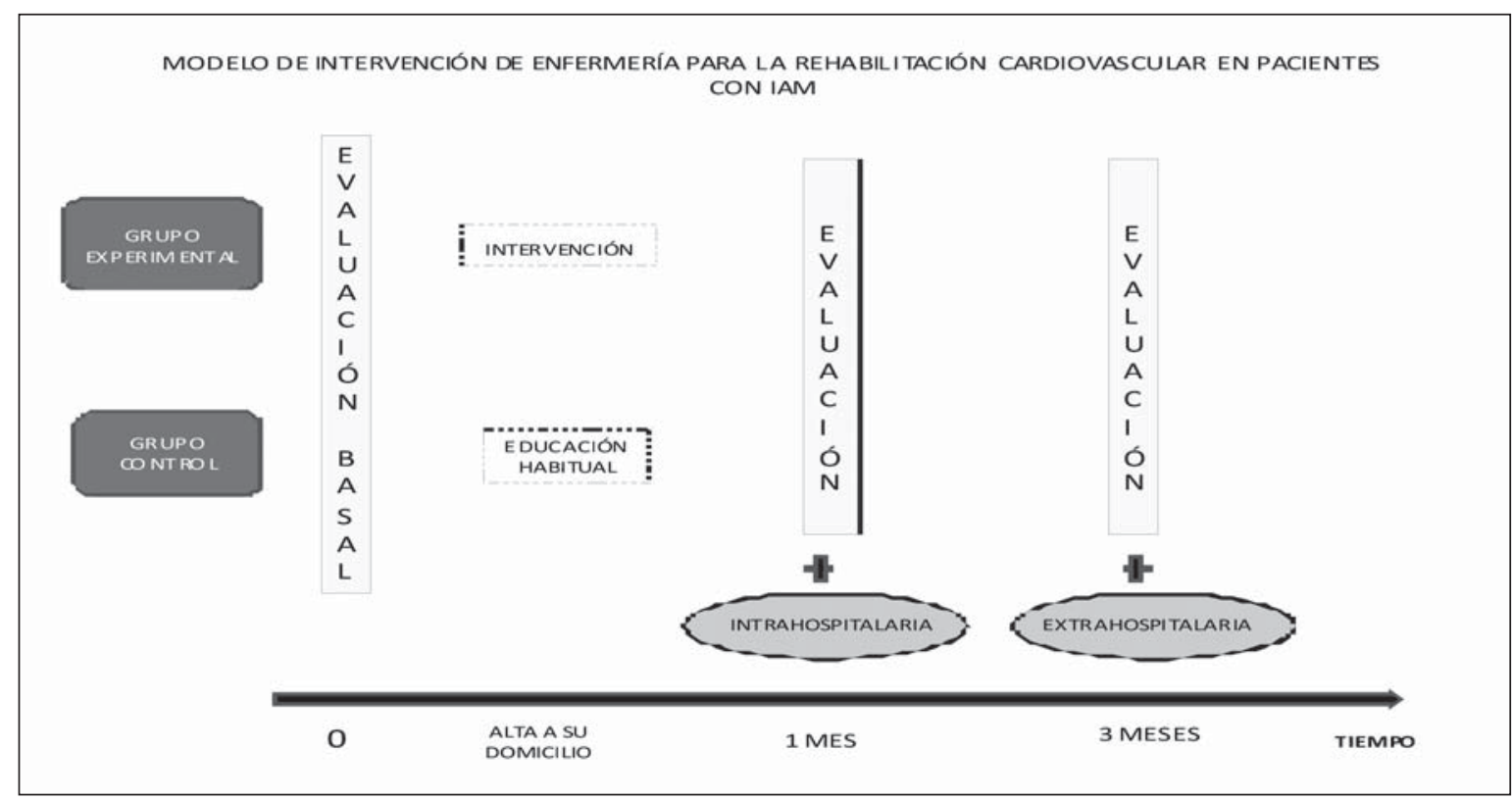

Para el análisis estadístico se utilizaron los software estadísticos SPSS versión 12, SAS versión 9.2 e InfoStat 2012. Se realizó un análisis descriptivo univariado de todas las variables en estudio, previo a la intervención. Para la comparación de las respuestas antes y después, en cada grupo, se utilizó el test t de Student para grupos pareados en el caso de variables numéricas y el test Mc Nemar (test de Bowker) para variables categóricas. Para la comparación del efecto de la intervención entre los grupos control y experimental se utilizó el test t de Student para las variables numéricas y el test chi-cuadrado (test exacto de Fisher) para las variables categóricas. Cuando el supuesto de normalidad en las variables numéricas no se verificó se utilizó el correspondiente test no paramétrico. Se utilizó un nivel de significancia del 0,05.

Para la realización de esta investigación fue necesario realizar una solicitud de evaluación al comité de ética del Hospital Guillermo Grant Benavente de Concepción, requisito establecido por esta Institución para la ejecución de toda investigación que se aplique en el establecimiento, solicitando consentimiento informado a todos los parti- cipantes en el estudio.

\section{RESULTADOS}

Los participantes fueron en un 63\% de sexo masculino y un $37 \%$ femenino, presentaban un promedio de edad de $63 \pm 12$ años, agrupados preferentemente en el grupo de adulto maduro con un $52 \%$, que comprende a aquellos entre 46 y 65 años, seguido por los pacientes mayores de 65 años que corresponde a adulto mayor, con un $40 \%$. En cuanto a la actividad, un $73 \%$ se mantenía activo, es decir desempeñaba algún trabajo remunerado, mientras que un $27 \%$ inactivo, es decir cesante o jubilado. Un $60 \%$ de los participantes en el estudio se encontraba con pareja, y un $40 \%$ sin pareja, este último grupo corresponde a aquellos pacientes viudos, separados o solteros.

En las variables clínicas se encontró que la media del peso fue de $81 \pm 12 \mathrm{~kg}$, el que varió de 60 a $117 \mathrm{~kg}$, por lo tanto, al realizar el cálculo del índice de masa corporal (IMC) dio como resultado una media de 27,6 $\pm 2,7$, 
lo que indica que la mayor parte del grupo en estudio tenía sobrepeso (67\%). En cuanto a la circunferencia de cintura se obtuvo un valor de 103,6 $\pm 12,3 \mathrm{~cm}$, con un rango de $78 \mathrm{y}$ $128 \mathrm{~cm}$. Sobre los parámetros hemodinámicos, la media de la frecuencia cardiaca fue de 77,5 $\pm 15,9$ lmp, con una mínima de 50 y un máximo de $128 \mathrm{lpm}$, las medias de presión arterial sistólica y diastólica se mantuvieron dentro de rangos normales.

En la incidencia de FR cardiovascular la hipertensión arterial ocupó el primer lugar con un $76 \%$, seguida de la dislipidemia con un $56 \%$ y los antecedentes familiares de enfermedad coronaria con un 53\%, actividad física con un $21 \%$ y la obesidad $25 \%$ de los pacientes. Es importante destacar que el 29\% de la población en estudio padecía simultáneamente de hipertensión arterial y diabetes mellitus, lo que aumenta la incidencia de padecer algún evento coronario. En lo que respecta a los FR cardiovascular y la incidencia de éstos se encontró que un 32\% de los pacientes presentaba 5 factores de riesgo, pero al realizar este análisis por sexo se destaca que en el sexo femenino el mayor porcentaje de mujeres presentaba de 5 a 9 factores de riesgo, a diferencia de lo que ocurre con el sexo masculino que presentaban desde 2 hasta 7 factores de riesgo.

Las características clínicas del grupo experimental y grupo control, previo a la intervención de enfermería, indican que ambos grupos fueron homogéneos en cuanto a la edad, días hospitalizados y valores demográficos y hemodinámicos. En lo relacionado a los factores de riesgo (Tabla 1), sólo se encontró una diferencia significativa en los antecedentes familiares $(\mathrm{p}=0,0178)$, debido a que en el grupo control hubo un 63\% de pacientes con antecedentes coronarios en su familia, mientras que en el grupo experimental sólo un $35 \%$. En el resto de los factores no hubo diferencias significativas.

Tabla 1. Factores de riesgo cardiovascular grupo control y experimental previo a la intervención.

\begin{tabular}{lccccc}
\hline & Control $(\mathrm{N}=32)$ & \multicolumn{3}{c}{ Experimental $(\mathrm{N}=31)$} & \\
\cline { 2 - 5 } VARIABLE & Frecuencia & $\%$ & Frecuencia & $\%$ & $\mathrm{p}$ \\
\hline Hipertensión arterial & 24 & $75 \%$ & 24 & $77 \%$ & 0,8217 \\
Diabetes Mellitus & 8 & $25 \%$ & 12 & $39 \%$ & 0,2425 \\
Antecedentes familiares & 20 & $63 \%$ & 11 & $35 \%$ & 0,0178 \\
Obesidad & 8 & $25 \%$ & 8 & $26 \%$ & 0,9414 \\
Dislipidemia & 16 & $50 \%$ & 19 & $61 \%$ & 0,3673 \\
Tabaquismo & 13 & $41 \%$ & 15 & $48 \%$ & 0,5354 \\
Alcohol & 9 & $28 \%$ & 12 & $39 \%$ & 0,3729 \\
Actividad física & 6 & $19 \%$ & 7 & $23 \%$ & 0,7072 \\
\hline
\end{tabular}

En el análisis de las variables peso, circunferencia de cintura y parámetros hemodinámicos, frecuencia cardiaca y presión arterial, en el grupo control sexo femenino encontramos que al mes existe una diferencia mínima en todas las variables medidas, sin embargo al realizar la comparación de medias a los 3 meses, tenemos diferencias significativas principalmente en peso y circunferencia de cintura. El IMC, como es un parámetro que se deriva del peso corporal del paciente, demuestra una diferencia significativa entre 
evaluación basal y a los 3 meses. A pesar que en la frecuencia cardiaca se aprecia una mayor diferencia desde la evaluación basal a los
3 meses, no es significativo, lo mismo que ocurre con la presión arterial sistólica (Tabla 2).

Tabla 2. Comparación medición basal, 1 mes y 3 meses en grupo control de las variables clínicas.

\begin{tabular}{lccccc}
\hline VARIABLE & Basal & $\mathbf{1 ~ m e s}$ & $\mathbf{3}$ meses & Dif. & valor $\mathbf{p}$ \\
\hline Mujeres & & & & & \\
Peso & 76,9 & 76,2 & 76 & $-0,91$ & 0,0169 \\
Circunferencia cintura & 99,9 & 98,8 & 97,9 & -2 & 0,0006 \\
IMC & 27,53 & 27,25 & 27,19 & $-0,95$ & 0,0169 \\
Frecuencia cardiaca & 78,5 & 81,2 & 75,7 & $-2,73$ & 0,5511 \\
Presión arterial sistólica & 118,2 & 117,4 & 121,2 & 3 & 0,7145 \\
Presión arterial diastólica & 69,2 & 69,3 & 69,9 & 0,73 & 0,9204 \\
\hline Hombres & & & & & \\
Peso & 84,7 & 84 & 83,6 & $-2,83$ & 0,0032 \\
Circunferencia cintura & 105,3 & 104,2 & 103,9 & $-4,17$ & 0,0001 \\
IMC & 27,53 & 27,29 & 27,2 & $-0,84$ & 0,0032 \\
Frecuencia cardiaca & 77,5 & 78,7 & 79,6 & $-1,92$ & 0,2004 \\
Presión arterial sistólica & 121,4 & 121,1 & 121,9 & $-6,33$ & 0,954 \\
Presión arterial diastólica & 71,2 & 71,5 & 71,9 & $-4,83$ & 0,6441 \\
\hline
\end{tabular}

En el grupo control sexo masculino se da algo similar a lo que ocurre en los pacientes de sexo femenino, ya que es significativa la diferencia entre peso y circunferencia de cintura, sin embargo en los parámetros hemodinámicos, aunque se aprecia una mayor diferencia en sus medias, comparando la evaluación basal y la de los 3 meses, no es significativa. En los varones del grupo control se observó que el IMC se mantiene con una variación que, aunque es pequeña, es significativa $(\mathrm{p}=0,0032)$, sin embargo estas medias demuestran que los pacientes se encuentran en su mayoría del grupo con sobrepeso, al igual que en las mujeres (Tabla 2).

Al comparar en el grupo control los FR de los pacientes con IAM, antes y después de la aplicación de los instrumentos de evaluación, se obtiene como resultado que se producen diferencias significativas en todos ellos, como son disminución en el hábito tabáquico, alcohol y aumento de la actividad física. Los pacientes, a pesar que la educación o entrega de indicaciones en el hospital es muy limitada, mantienen los cuidados necesarios para modificar los FR cardiovascular, no obstante las diferencias son pequeñas éstas son significativas (Tabla 3 ).

Tabla 3. Comparación de los factores de riesgo cardiovascular en grupo control.

\begin{tabular}{lccccc}
\hline & Antes & \multicolumn{5}{c}{ Después } \\
\cline { 2 - 6 } VARIABLE & Frecuencia & $\%$ & Frecuencia & $\%$ & $\mathbf{p}$ \\
\hline Tabaquismo & 13 & 41 & 8 & 25 & 0,0253 \\
Alcohol & 9 & 28 & 3 & 9 & 0,0143 \\
Actividad física & 7 & 22 & 20 & 63 & 0,0003 \\
\hline
\end{tabular}


En el grupo experimental al realizar una comparación de las variables peso, circunferencia de cintura e IMC, en mujeres, se observó que entre la evaluación basal y el mes posterior a la intervención de enfermería hay variaciones en los valores de todas las variables, sin embargo al realizar la diferencia a los 3 meses son significativas: el peso, circunferencia de cintura e IMC. Es importante señalar que al cabo de los tres meses, no hay pacientes en la categoría de obesas en el grupo experimental, todas han disminuido de peso. Los parámetros hemodinámicos presentan diferencias significativas en la presión arterial tanto sistólica como diastólica, lo que está asociado a la disminución del peso. En cuanto a la frecuencia cardiaca también se observa una diferencia, pero ésta no es significativa. Mientras que en los pacientes de sexo masculino los valores obtenidos son similares a los del grupo experimental sexo femenino, sobre todo en lo relacionado a peso, circunferencia de cintura e IMC, sin embargo en los parámetros hemodinámicos sólo se encuentran diferencias significativas en la presión arterial sistólica $(\mathrm{p}=0,0560)$ (Tabla 4$)$.

Tabla 4. Comparación medición basal, 1 mes y 3 meses en grupo experimental de las variables clínicas.

\begin{tabular}{lccccc}
\hline VARIABLE & Basal & 1 mes & 3 meses & Dif. & valor p \\
\hline Mujeres & & & & & \\
Peso & 71,8 & 70,3 & 68,9 & $-1,1$ & $<0,0001$ \\
Circunferencia cintura & 97,3 & 95,1 & 93,1 & $-1,43$ & $<0,0001$ \\
IMC & 27,29 & 26,71 & 26,21 & $-1,36$ & $<0,0001$ \\
Frecuencia cardiaca & 77,7 & 76 & 75,8 & 2,1 & 0,578 \\
Presión arterial sistólica & 126,3 & 121,8 & 119,9 & 0,48 & 0,0003 \\
Presión arterial diastólica & 73,3 & 71,8 & 68,4 & 0,62 & 0,0179 \\
\hline Hombres & & & & & \\
Peso & 86 & 85,2 & 82,6 & $-3,42$ & $<0,0001$ \\
Circunferencia cintura & 107,7 & 106,2 & 103,4 & $-4,32$ & $<0,0001$ \\
IMC & 28,02 & 27,74 & 26,92 & $-1,74$ & $<0,0001$ \\
Frecuencia cardiaca & 76,8 & 76,2 & 74,3 & $-2,53$ & 0,8421 \\
Presión arterial sistólica & 121,8 & 117,6 & 118 & $-3,79$ & 0,056 \\
Presión arterial diastólica & 70,6 & 68,7 & 69,3 & $-1,37$ & 0,5281 \\
\hline
\end{tabular}

En la Tabla 5 se observan los datos del grupo experimental en relación a los FR cardiovascular, las 3 variables que se evaluaron son las que más se asocian a morbimortalidad cardiovascular: hábito tabáquico, consumo de alcohol y actividad física. En cuanto a los resultados obtenidos entre el antes y el después de la intervención, todas son significativas, ya que en cada una de ellas hubo modificaciones por parte de los pacientes. En el hábito tabáquico, del $48 \%$ de pacientes que fumaba posterior a los 3 meses, sólo un $6 \%$ de ellos se mantuvo con este hábito; el consumo de alcohol disminuyó de un 39 a un 13\%, lo que dio un $\mathrm{p}=0,0047$. Por último, la actividad física aumento de un 19 a un 55\% de pacientes que realizaba actividad física. Esta actividad no está enfocada a la que se realiza en un gimnasio, sino a la que el paciente pueda realizar en su hogar en forma controlada.

Para el análisis de las diferencias de grupos control y experimental, se consideró la evaluación basal y la de los 3 meses posintervención de enfermería, ya que es donde se 
Tabla 5. Comparación de los factores de riesgo cardiovascular grupo experimental.

\begin{tabular}{lccccc}
\hline & Antes & \multicolumn{5}{c}{ Después } \\
\cline { 2 - 6 } VARIABLE & Número & $\%$ & Número & $\%$ & \multicolumn{1}{c}{$\mathbf{p}$} \\
\hline Tabaquismo & 15 & 48 & 2 & 6 & 0,0003 \\
Alcohol & 12 & 39 & 4 & 13 & 0,0047 \\
Actividad física & 6 & 19 & 17 & 55 & 0,0009 \\
\hline
\end{tabular}

aprecian las mayores diferencias. Al realizar la comparación de las diferencias entre el grupo control y experimental, en pacientes de sexo femenino y masculino, se observó que para el peso y circunferencia de cintura hay diferencias significativas entre ambos grupos, por lo tanto, en el IMC también $(\mathrm{p}<0,0001)$. Lo que muestra que la intervención educativa orientada al cuidado de enfermería es efectiva para disminuir estas variables en los pacientes del estudio. En lo que respecta a los parámetros hemodinámicos se encontraron diferencias significativas en la presión arterial sistólica sólo en las mujeres (Tabla 6).

Tabla 6. Diferencia de las diferencias de las medias de las variables clínicas, entre los grupos control y experimental.

\begin{tabular}{lccccccc}
\hline \multicolumn{7}{c}{ Control } & \multicolumn{2}{c}{ Experimental } & & & \\
\hline VARIABLE & Basal & 3 meses & Basal & 3 meses & Dif. control & Dif. exper. & p \\
\hline Mujeres & & & & & & & \\
Peso & 76,9 & 76 & 71,8 & 68,9 & $-0,91$ & $-2,83$ & 0,0002 \\
Circunferencia cintura & 99,9 & 97,9 & 97,3 & 93,1 & -2 & $-4,17$ & 0,0323 \\
IMC & 27,53 & 27,19 & 27,29 & 26,21 & $-0,34$ & $-1,08$ & $<0,0001$ \\
Frecuencia cardiaca & 78,5 & 75,7 & 77,7 & 75,8 & $-2,73$ & $-1,92$ & 0,913 \\
Presión arterial sistólica & 118,2 & 121,2 & 126,3 & 119,9 & 3 & $-6,33$ & 0,0065 \\
Presión arterial diastólica & 69,2 & 69,9 & 73,3 & 68,4 & 0,73 & $-4,83$ & 0,2465 \\
\hline Hombres & & & & & & & \\
Peso & 84,7 & 83,6 & 86 & 82,6 & $-1,1$ & $-3,42$ & 0,0001 \\
Circunferencia cintura & 105,3 & 103,9 & 107,7 & 103,4 & $-1,43$ & $-4,32$ & 0,0004 \\
IMC & 27,53 & 27,2 & 28,02 & 26,92 & $-0,33$ & $-1,1$ & $<0,0001$ \\
Frecuencia cardiaca & 77,5 & 79,6 & 76,8 & 74,3 & 2,1 & $-2,53$ & 0,2431 \\
Presión arterial sistólica & 121,4 & 121,9 & 121,8 & 118 & 0,48 & $-3,79$ & 0,2225 \\
Presión arterial diastólica & 71,2 & 71,9 & 70,6 & 69,3 & 0,62 & $-1,37$ & 0,565 \\
\hline
\end{tabular}

En relación a los FR cardiovascular, entre grupo control y experimental (Tabla 7), se observó que hay diferencias significativas en el hábito tabáquico, ya que en el grupo control dejó de fumar el $38 \%$ de los pacientes, mientras que en el grupo experimental lo dejó de hacer un $87 \%$ de los pacientes, por lo tanto la intervención orientada al cuidado de pacientes demuestra que tiene un efecto positivo sobre el control y modificación de este factor de riesgo cardiovascular.

En lo que respecta al consumo de alcohol, 
Tabla 7. Diferencias de los FR cardiovascular entre grupo control y grupo experimental.

\begin{tabular}{lccccccr}
\hline & \multicolumn{9}{c}{ Control } & \multicolumn{3}{c}{ Experimental } & & \\
\cline { 2 - 6 } VARIABLE & Antes & Después & $\%$ & Antes & Después & $\%$ & p \\
\hline Tabaquismo & 13 & 5 & 38 & 15 & 13 & 87 & 0,0163 \\
Alcohol & 9 & 6 & 67 & 12 & 8 & 67 & $>0,9999$ \\
Actividad física & 19 & 7 & 27 & 7 & 17 & 71 & 0,0019 \\
\hline
\end{tabular}

no hubo diferencias significativas, a pesar que el número de pacientes que consumía alcohol en ambos grupos disminuyó, lo que es importante destacar porque el grupo que se mantenía consumiendo también disminuyó en la cantidad de alcohol ingerido. En cuanto a la actividad física, el aumento en la práctica de actividad física de los pacientes del grupo experimental fue significativo, ya que se incrementó en un 71\% en el grupo experimental comparado con un $27 \%$ del grupo control $(p=0,0019)$. Los resultados obtenidos en torno a los factores de riesgo cardiovascular modificables muestran lo importante que es una intervención orientada al cuidado de enfermería en esta etapa de la rehabilitación.

\section{DISCUSIÓN Y CONCLUSIÓN}

En la población chilena no se encuentran reportes en la literatura respecto a una intervención educativa orientada al cuidado de enfermería en pacientes que han sufrido un IAM, y de algún seguimiento posterior a su egreso hospitalario. En esta investigación que se propuso estudiar el efecto de esta intervención comparándola con la entrega de indicaciones habitual, se evidenció la importancia que tiene una intervención en la recuperación de los pacientes.

En cuanto a las características biosociodemográficas de los 63 pacientes que participaron en esta investigación, la edad promedio fue de 63 años, con mayor prevalencia de hombres, lo cual coincide con la literatura donde se señala que el ser mayor de 55 años y ser varón aumentan el riesgo cardiovascular y son características utilizadas para estratificar el riesgo coronario (13). Más allá de la edad biológica, se debe tener en cuenta la existencia de comorbilidades y condiciones asociadas, como deterioro cognitivo y funcional y la dependencia física. El riesgo cardiovascular en mujeres, al igual que en los varones, puede reducirse dejando de fumar, realizando actividad física, evitando el sobrepeso y manteniendo cifras de presión arterial estables. Esto coincide con esta investigación, ya que se consideraron los FR y edad en las mujeres, los que son mayores que en los hombres, y tienen mayor cantidad de FR cardiovascular.

En el ámbito laboral, se encontró que el $73 \%$ de la muestra se encontraba activa laboralmente; por lo que es importante señalar que el estrés relacionado con el trabajo (exigencias psicológicas excesivas, falta de apoyo social y presión) es FR cardiovascular principalmente en varones (14). Los estudios sobre mujeres en relación a este tema son pocos y no permiten extraer conclusiones confiables. En las últimas décadas la evidencia indica (15) que el aumento del riesgo cardiovascular empieza a aparecer a edades muy tempranas debido a la exposición de las personas a los FR. Dentro de las comorbilidades que precedían el evento isquémico en la población en estudio, se observó que un $76 \%$ de los pacientes sufría de HTA, correspondiendo a un importante FR de enfermedad coronaria. En 
una serie de estudios epidemiológicos (16, 17), la presión arterial elevada se ha identificado como un importante elemento, junto a la coexistencia de otros como: tabaquismo, colesterol elevado, diabetes mellitus, historia familiar de enfermedad cardiovascular prematura, lo que aumenta de forma importante el riesgo asociado a una ligera elevación de la presión arterial (17).

Aproximadamente un $20-30 \%$ de los pacientes con enfermedad coronaria tiene diabetes conocida, y por lo menos la misma proporción tiene diabetes no diagnosticada o una alteración de la tolerancia a la glucosa. El Euro Heart Survey ha revelado que el 37\% de los pacientes tenía diabetes establecida o de nuevo diagnóstico (18). La diabetes mellitus es un predictor independiente de mortalidad, por lo tanto, los pacientes diabéticos tienen el doble riesgo de muerte (19). La evidencia existente se relaciona directamente con la población de este estudio, un 32\% de los pacientes padece de diabetes mellitus, y al realizar el análisis por sexo, un $48 \%$ de las mujeres tiene esta patología, por lo que el riesgo en este grupo se ve aumentado. Además, se destaca que un $43 \%$ de ellas sufre de esta misma patología e hipertensión arterial. Por lo tanto, los diabéticos son pacientes de alto riesgo y, como tales, necesitan un manejo invasivo y farmacológico agresivo, además de un enfoque integral para la prevención secundaria que debe incluir el tratamiento farmacológico y cambios en el estilo de vida.

Los FR en los participantes de este estudio fue realizado en base a lo que ellos señalaban en la entrevista, los porcentajes obtenidos son los siguientes: un $44 \%$ tenía hábito tabáquico; un 33\% consumía alcohol, pero de éstos sólo el 5\% eran considerados alcohólicos, ya que los demás pacientes bebían alcohol en forma ocasional; un $25 \%$ era obeso; $56 \%$ dislipidémico y sólo un $21 \%$ de los participantes realizaba algún tipo de actividad física. El hábito tabáquico es uno de los de mayor incidencia en este grupo de estudio, y es una causa establecida del 50\% de todas las muer- tes evitables de fumadores por enfermedad coronaria. Según la estimación del sistema SCORE, el riesgo de eventos cardiovasculares mortales a 10 años se multiplica por 2 en los fumadores $(20,21)$. Tradicionalmente, el tabaquismo afectaba a los varones, pero en los últimos años, las mujeres han alcanzado o sobrepasado las tasas de tabaquismo de los varones (22). En la población de este estudio se mantiene con lo tradicional, ya que un $55 \%$ de los hombres y un 26\% de las mujeres eran fumadores. Debido a la elevada incidencia de la enfermedad coronaria y la amplia exposición ambiental al humo del tabaco, se esperan beneficios importantes con la reducción del humo de tabaco en el ambiente, ya que la evidencia muestra que el tabaquismo pasivo aumenta el riesgo de enfermedad coronaria. Una persona no fumadora que vive con un fumador tiene un $30 \%$ más riesgo de ECV (23).

La obesidad también está presente en los pacientes que participan en esta investigación, en un 25\% del total; sin embargo al realizar el análisis por sexo, el 17\% de las mujeres es obesa en comparación a un 30\% de los varones. Se considera a la obesidad junto al sobrepeso un factor de riesgo de muerte de la $\operatorname{ECV~}(24,25)$. La obesidad se ha convertido en una epidemia mundial, tanto en niños como en adultos. Algunos estudios prospectivos han encontrado evidencia de una mayor asociación entre la adiposidad abdominal y la enfermedad coronaria que entre el IMC y la EC en mujeres (26), pero no en los varones.

La actividad física se asocia con una disminución de la mortalidad cardiovascular. El estilo de vida sedentario es uno de los principales FR de enfermedad coronaria. Se recomiendan la actividad física y el ejercicio aeróbico como herramientas importantes para la prevención primaria y secundaria (27). La actividad física también tiene un efecto positivo en muchos de los FR establecidos, porque previene o retrasa el desarrollo de hipertensión y reduce la presión arterial en 
pacientes hipertensos, aumenta las concentraciones de cHDL, ayuda a controlar el peso y reduce el riesgo de diabetes mellitus (28). Esto se relaciona con lo obtenido por el grupo experimental, ya que del total pacientes sólo un $21 \%$ realizaba actividad física, previo al evento coronario, por lo tanto un $79 \%$ de los pacientes era sedentario y no conocían la importancia de la actividad física. Al realizar este análisis por sexo, es importante destacar que un $9 \%$ de las mujeres realizaba algún tipo de actividad física en comparación a un $27 \%$ de los hombres. Posterior a la intervención educativa, el número de pacientes que comenzó a realizar actividad física aumento significativamente a un $71 \%$, lo que además mejoro sus cifras de presión arterial y frecuencia cardiaca.

Sobre el impacto de la intervención educativa de Enfermería aplicada en este estudio, se encontraron modificaciones significativas en los factores de riesgo modificables, al igual que en el estudio de Scalzi (29), que estudio la relación entre la participación en un programa educacional organizado, con mediciones $1^{\circ}, 3^{\circ}, 6^{\circ}, 12^{\circ}, 18^{\circ}$ y $24^{\circ}$ meses posalta. Se concluye que la retención de información es limitada durante la fase aguda de la enfermedad, pero la instrucción continua en visitas posteriores parece mejorar el conocimiento y la adherencia. En todas las áreas, excepto en el cese de fumar el grupo experimental demostró mayor adherencia y conocimiento. Por lo tanto, y de acuerdo a los resultados obtenidos en el estudio hubo modificaciones importantes en los pacientes en relación a su autocuidado, sin embargo es fundamental continuar con este apoyo por un período más prolongado y continuo.

En esta investigación se pudo evidenciar que la intervención educativa, seguida de una evaluación al mes y a los tres meses, ocasionó modificaciones en los FR, lo que se asoció a un mejor estilo de vida. Entre grupo control y experimental, hubo diferencias significativas en el peso, circunferencia de cintura, IMC y presión arterial sistólica en los pacientes de sexo femenino, mientras que en los pacientes de sexo masculino se observaron estas diferencias sólo en el peso, IMC y circunferencia de cintura. En lo que se refiere al hábito tabáquico, alcohol y actividad física, se encontró diferencias estadísticamente significativas en el tabaco y la actividad física.

Los programas de prevención coordinados por personal de enfermería son efectivos en distintos contextos de la práctica clínica. En ensayos clínicos aleatorizados sobre prevención secundaria se han evaluado distintos modelos de manejo llevados a cabo por enfermeras, y se han observado beneficios significativos en relación con FR, tolerancia al ejercicio, control de la glucosa y uso apropiado de la medicación, junto con una reducción en la incidencia de eventos y mortalidad cardiacos, una mayor regresión de la aterosclerosis coronaria y una mejor percepción del paciente sobre su salud frente a los cuidados convencionales (30). Uno de los puntos fuertes del estudio fue que demostró la factibilidad de este tipo de programas en hospitales y consultas de atención primaria, este estudio muestra que no es necesario un centro especializado para poder realizar rehabilitación a los pacientes coronarios, y que en Chile se podrían implementar medidas en los hospitales, considerando que sólo cuenta con 8 centros a nivel nacional.

A pesar del éxito de las intervenciones, se confirma el principio básico de la necesidad de un contacto continuo para lograr cambios en el estilo de vida. Hacen falta más estudios para determinar el formato óptimo de las intervenciones destinadas a lograr una reducción perdurable del riesgo, y el grado de intensidad y adaptación de las intervenciones a personas con diferentes necesidades y niveles de riesgo en distintos contextos sanitarios y comunitarios. Aunque hay indicios de que estos modelos son coste-efectivos (31), aún deben evaluarse adecuadamente, al igual que las medidas para conseguir una mayor conciencia sobre el riesgo y los cambios en el comportamiento en el contexto de la pre- 
vención primaria.

La RC se considera una intervención coste-efectiva tras un evento coronario; mejora el pronóstico porque reduce el número de hospitalizaciones sucesivas y los gastos sanitarios, a la vez que prolonga la vida. Si bien los componentes fundamentales y los objetivos de la RC están estandarizados y documentados, la estructura y el tipo de las unidades de rehabilitación pueden variar de un país a otro. La tradición del propio sistema de salud y las consideraciones económicas tienen un papel importante. Como resultado de las intervenciones de RC se espera mayor control de los síntomas y estabilidad clínica, una reducción del riesgo cardiovascular, mayor adherencia a la medicación y mejor perfil de comportamiento. El éxito de los esfuerzos en prevención secundaria y rehabilitación depende de la atención y el apoyo que reciba el individuo, lo que incluye un examen exhaustivo de su estado clínico, no sólo de la función $\mathrm{CV}$, sino también de la comorbilidad y los factores psicosociales. Se reconoce, además, la necesaria comunicación con la atención primaria que sería un factor relevante para dar continuidad a un programa de intervención a largo plazo. Este trabajo en red y una buena información son determinantes para el éxito de una tarea como es la RC.

Sobre la comparación de los resultados de la intervención orientado al cuidado de enfermería en la RC, con la educación habitual otorgada a los pacientes post IAM, se destaca que la intervención educativa orientada al cuidado de enfermería en la RC puede modificar aquellos factores que inciden en el evento coronario, por lo tanto, de esta forma disminuye la morbilidad y mejora su calidad de vida.

Finalmente, fue posible evaluar la efectividad de una intervención de enfermería educativa orientada al Cuidado en la Rehabilitación Cardiovascular de pacientes post infarto agudo al miocardio. Aún cuando los resultados de este estudio dan cumplimiento con el objetivo de la investigación, surgen nuevas preguntas derivadas de estos hallazgos que ameritan una ampliación de los fenómenos de estudio intentando superar las siguientes limitantes. Para el profesional de enfermería, todo lo antes descrito se convierte en un gran reto dado que permite enfocar el actuar de Enfermería en la implementación de estrategias de intervención para el cuidado de la salud cardiovascular de las personas con un IAM y dar respuesta a los problemas planteados en la práctica clínica asociados a los comportamientos de adherencia y a sus factores intervinientes. También es un desafío incorporar la atención primaria en esta tarea, ya que, este espacio puede dar continuidad a una intervención que debe comenzar en el Hospital, pero que difícilmente, sin el apoyo de la enfermería comunitaria, para la realización de las visitas domiciliarias es muy difícil de proyectar a largo plazo.

\section{REFERENCIAS}

1. Murray CJ, López AD. Alternative projections of mortality and disability by cause 1990-2020: Global Burden of Disease Study. Lancet. 1997; 349: 1498-504.

2. Hamm C, Bassand J, Agewall S, Bax J, Boersman E, Bueno $\mathrm{H}$, et al. Guía de práctica clínica de la Sociedad Europea de Cardiología (ESC) para el manejo del síndrome coronario agudo en pacientes sin elevación persistente del segmento ST. Grupo de Trabajo para el manejo del SCA en pacientes sin elevación persistente del segmento ST de la ESC. Rev Esp Cardiol. 2012; 65(2): 173.e1-e55.

3. Dawber TR, Kannel WB. The Framingham study. An epidemiological approach to coronary heart disease. Circulation, 1966; 34(4): 553-5.

4. Ministerio de Salud (MINSAL), Gobierno de Chile. Foros de Salud: Desafíos y propuestas ciudadanas Construyamos Juntos una Mejor Salud para Todos y 
Todas al 2020. Santiago, Chile: MINSAL; 2010.

5. O’ Donnell CJ, Elousa R. Factores de riesgo cardiovascular. Perspectivas derivadas del Framingham Heart Study. Rev Esp Cardiol. 2008; 61: 299-310.

6. León M, Mazón P, Marcos E, García E. Temas de actualidad en prevención cardiovascular y rehabilitación cardiaca. Rev Esp Cardiol. 2009; 62(Supl 1): 4-13

7. O'Connor CG, Buring JE, Yusuf S, Goldhaber SZ, Olmstead BA, Paffenbarger RS et al. An overview of randomized trials of rehabilitation with exercise after myocardial infarction. Circulation. 1989; 80(234): 244.

8. WHO Expert Committee on Rehabilitation of Patients with Cardiovascular Diseases/World Health Organization. World Health Organization Rehabilitation of patients with cardiovascular disease. World Health Organ Tech Rep Ser. Geneve: OMS; 1964.

9. Winslow EH, Weber TM. Rehabilitation of the cardiac patient. Progressive exercise to combat the hazard of bed rest. Am J Nurs. 1980; 80(3): 440-443.

10. Scalzi CC, Burke LE, Greenland S. Evaluation of an inpatient educational program for coronary patients and families. Heart Lung. 1980; 9(5): 846-53.

11. Sánchez R, Echeverry J. Validación de escalas de medición en salud. Revista de salud pública. 2004; 6(3): 302-318.

12. Fernández C, Baptista P. Metodología de la investigación. 4a ed. Distrito Federal, México: McGraw-Hill Interamericana; 2008.

13. Rugulies R. Depression as a predictor for coronary heart disease. a review and meta-analysis. Am J Prev Med. 2002; 23(1): 51-61.

14. Steenland K. Risk assessment for heart disease and workplace ETS exposure among nonsmokers. Environ Health Perspect. 1999; 107 Suppl 6: 859-63.226 prev.
15. Chow CK, Jolly S, Rao-Melacini P, Fox KA, Anand SS, Yusuf S. Association of diet, exercise, and smoking modification with risk of early cardiovascular events after acute coronary syndromes. Circulation. 2010; 121: 750-8.

16. Steenland K. Risk assessment for heart disease and workplace ETS exposure among nonsmokers. Environ Health Perspect. 1999;107 Suppl 6: 859-63. 226 prev.

17. Kavanagh T, Mertens DJ, Hamm LF, Beyene J, Kennedy J, Corey P, et al. Prediction of long-term prognosis in 12169 men referred for cardiac rehabilitation. Circulation. 2002; 106: 666-71.

18. Kavanagh T, Mertens DJ, Hamm LF, Beyene J, Kennedy J, Corey P, et al. Peak oxygen intake and cardiac mortality in women referred for cardiac rehabilitation. J Am Coll Cardiol. 2003; 42(12): 2139-43.

19. Hambrecht R, Walther C, Mobius-Winkler S, Gielen S, Linke A, Conradi K, et al. Percutaneous coronary angioplasty compared with exercise training in patients with stable coronary artery disease: a randomized trial. Circulation. 2004;109 (11):1371-8.

20. O'Connor CM, Whellan DJ, Lee KL, Keteyian SJ, Cooper LS, Ellis SJ, et al. Efficacy and safety of exercise training in patients with chronic heart failure: HF-ACTION randomized controlled trial. JAMA 2009; 301(14): 1439-50.

21. Freiberg MS, Pencina MJ, D’Agostino RB, Lanier K, Wilson PW, Vasan RS. BMI vs. waist circumference for identifying vascular risk. Obesity (Silver Spring). 2008; 16(2): 463-9376

22. Warren TY, Barry V, Hooker SP, Sui X, Church TS, Blair SN. Sedentary behaviors increase risk of cardiovascular disease mortality in men. Med Sci Sports Exerc. 2010; 42(5): 879-85.

23. Stringhini S, Sabia S, Shipley M, Brunner E, Nabi H, Kivimaki M, et al. Association of socioeconomic position with health 
behaviors and mortality. JAMA. 2010; 303(12): 1159-66.

24. Rozanski A, Blumenthal JA, Davidson KW, Saab PG, Kubzansky L. The epidemiology, pathophysiology, and management of psychosocial risk factors in cardiac practice: the emerging field of behavioral cardiology. J Am Coll Cardiol. 2005; 45(5): 637-51.

25. Whooley MA, de Jonge P, Vittinghoff E, Otte C, Moos R, Carney RM, et al. Depressive symptoms, health behaviors, and risk of cardiovascular events in patients with coronary heart disease. JAMA. 2008; 300(2): 2379-88.

26. Steptoe A, Marmot M. The role of psychobiological pathways in socio-economic inequalities in cardiovascular disease risk. Eur Heart J. 2002; 23(1): 13-25.

27. Pinson A. Educación del paciente cardiovascular en los programas de rehabilitación cardiaca Educación del paciente cardiovascular en los programas de rehabilitación cardiaca. Rev mex, enferm. cardiol. 2000; 8(1-4): 25-30.

28. Piepoli MF, Corrà U, Benzer W, Bjarna-
son-Wehrens B, Dendale P, Gaita D, et al. Secondary prevention through cardiac rehabilitation: from knowledge to implementation. A position paper from the Cardiac Rehabilitation Section of the European Association of Cardiovascular Prevention and Rehabilitation. Eur J Cardiovasc Prev Rehabil. 2010; 17(1): 1-17.

29. Scalzi CC, Burke LE, Greenland S. Evaluation of an inpatient educational program for coronary patients and families. Heart Lung. 1980; 9(5): 846-53.

30. Jolliffe JA, Rees K, Taylor RS, Thompson D, Oldridge N, Ebrahim S. Exercise-based rehabilitation for coronary heart disease. Cochrane Database Syst Rev. 2001; (1): CD001800.

31. Antman EM, Anbe DT, Armstrong PW, Bates ER, Green LA, Hand M, et al. ACC/ AHA guidelines for the management of patients with ST-elevation myocardial infarction-executive summary. A report of the American College of Cardiology/ American Heart Association Task Force on Practice Guidelines. J Am Coll Cardiol. 2004; 44(3): 671-719. 